\title{
Lignes directrices sur la prise en charge concertée des personnes qui ont le diabète sucré par des professionnels des soins oculovisuels
}

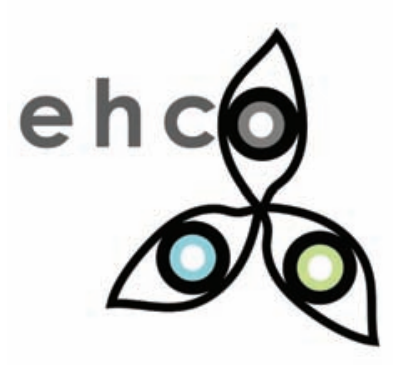

CONSEIL DE LA SANTÉ DE L'CEIL
DE L'ONTARIO

LE 23 SEPTEMBRE 2011

\section{Contexte}

Le diabète est une maladie dont l'incidence et la prévalence prennent rapidement de l'ampleur en Ontario (dépassant de façon spectaculaire les estimations globales de l'Organisation mondiale de la Santé) et qui pose un défi majeur en santé publique à de nombreux égards. ${ }^{1}$ Plus précisément, la rétinopathie diabétique constitue la cause la plus répandue de nouveaux cas de cécité légale chez les personnes en âge de travailler., ${ }^{2,12}$ La rétinopathie diabétique cause environ $12 \%$ des nouveaux cas de cécité et les personnes qui ont une rétinopathie diabétique sont de 25 à 29 fois plus susceptibles que la population générale de devenir aveugles dans les quatre ans. ${ }^{3,13}$ Jusqu'à $20 \%$ des patients chez lesquels on vient de diagnostiquer un diabète de type 2 (90\% des cas de diabète sont de type 2 ) présentent des signes de maladie oculaire reliée au diabète au moment du diagnostic et environ $5 \%$ auront besoin d'un traitement immédiat visant à les aider à éviter de perdre la vue. L'œil subit des changements reliés au diabète dans les sept ans suivant le diagnostic chez $50 \%$ des patients qui ont le diabète de type 2. Quinze ans plus tard, ce pourcentage atteint $85 \%$, et $25 \%$ ont besoin de traitement. ${ }^{3} \mathrm{La}$ totalité des patients qui ont le diabète de type 1 auront une maladie oculaire reliée au diabète 15 à 20 ans après le diagnostic., ${ }^{3,8}$ De plus, les changements vasculaires qui se produisent à l'intérieur de l'œil permettent de prévoir des changements vasculaires ailleurs dans le corps. ${ }^{6,7}$

La meilleure façon de traiter la perte de vision causée par la rétinopathie diabétique (et peut-être même de la prévenir) consiste à la découvrir à temps. ${ }^{4}$ Les données provenant des États-Unis et de l'Australie

\section{Le Conseil de la santé de l'œil de l'Ontario (CSOO)}

Il est clair depuis longtemps pour ceux qui s'occupent de la santé de l'œil en Ontario qu'il faut une tribune pour promouvoir la collaboration interprofessionnelle afin d'optimiser la prestation des soins oculovisuels et de diffuser ces concepts aux intervenants appropriés. Il y a environ six ans, l'Association des optométristes de l'Ontario et l'Association médicale de I'Ontario, Section de l'ophtalmologie, ont créé un Conseil des soins oculovisuels à cette fin. Le conseil est devenu depuis le Conseil de la santé de l'œil de l'Ontario (CSOO).

Le CSOO a tenu sa réunion inaugurale le 3 décembre 2010, dans le sillage des recommandations formulées le 31 mars 2010 par le Conseil consultatif sur la réglementation des professions de la santé (CCRPS) dans le « Rapport à la ministre de la Santé et des Soins de longue durée sur la collaboration interprofessionnelle au sein des professions des soins des yeux ». Ce rapport prévoyait un conseil constitué d'optométristes et d'ophtalmologistes conjuguant leurs efforts, semblable au modèle innovateur créé en Nouvelle-Écosse et basé sur l'assise existante déjà en Ontario.

Le CSOO a pour mandat d'appuyer la prestation de soins oculovisuels de qualité et accessibles à la population de l'Ontario en garantissant l'utilisation la plus efficace du continuum des professionnels des soins oculovisuels dans l'intérêt de la sécurité du patient, la qualité des soins et une prestation efficiente.

Le CSOO fera aussi entendre une voix unifiée sur les enjeux des soins oculovisuels au ministère de la Santé et des Soins de longue durée (MSSLD) et constituera un moyen d'établir des lignes directrices communes sur la collaboration dans le soin des patients et un contexte idéal pour la collaboration interprofessionnelle en dehors du cadre de la réglementation. Le Conseil est constitué de 14 personnes provenant des milieux de l'ophtalmologie et de l'optométrie qui représentent des organismes universitaires, politiques et de réglementation de chaque profession. Les deux professions se sont entendues sur une structure de gouvernance où les deux coprésidents, l'un étant optométriste et l'autre, ophtalmologiste, dirigent les réunions. Le CSOO approuve les points à l'ordre du jour par un vote majoritaire des deux tiers des voix. Le Conseil se réunit quatre fois par année et tient une fois par année une réunion prolongée à laquelle il invite tous les intervenants appropriés (c.-à-d. opticiens, industrie, INCA, médecins de famille, etc.). Le Conseil compte deux 
( La prévention de la cécité chez les personnes qui ont le diabète offre des possibilités de réduction des coûts et d'efficience sans pareilles. $\|$ y a peu de cas en soins de santé qui sont aussi évidents. \〉

JC Javitt, MD, MPH «Blindness: We Know What It Costs! Now What? » Symposium sur le coût de la cécité ${ }^{11}$

montrent malheureusement que $50 \%$ des personnes qui ont le diabète ne bénéficient pas d'un examen de la vue périodique. ${ }^{9,10} \mathrm{Ces}$ chiffres sont renversants lorsqu'on les extrapole aux quelque 3 millions de Canadiens qui vivent actuellement avec le diabète (dont le tiers ne savent pas qu'ils l'ont), total qui devrait passer à 3,7 millions d'ici à $2020^{5}$, selon les prévisions. Le taux de diabète est presque cinq fois plus élevé chez les Autochtones que chez les non-Autochtones et les premiers risquent plus que tout autre groupe ethnique au Canada de perdre la vue à cause du diabète et de ses complications oculaires. ${ }^{5}$
Les fournisseurs de soins oculovisuels ont un défi à relever pour gérer et coordonner le soin des patients qui ont le diabète. La prestation des soins oculovisuels doit prévoir l'utilisation rentable et efficiente des ressources afin de réduire au minimum la perte de vision évitable.

\section{Efficacité des méthodes actuelles d'évaluation de la rétinopathie diabétique (RD)}

L'évaluation joue un rôle important dans le dépistage précoce et l'intervention qui visent à empêcher la rétinopathie diabétique $(\mathrm{RD})$ d'évoluer. La basse vision et la cécité diminuent considérablement chez les personnes qui ont le diabète et reçoivent les soins recommandés. ${ }^{15} \mathrm{En}$ dépit de la grande efficacité à la fois clinique et financière de l'évaluation et du traitement de la rétinopathie diabétique, l'évaluation et l'observation des traitements posent toujours des problèmes. Beaucoup de personnes qui ont le diabète n'ont pas accès à des examens de la vue périodiques et les obstacles qui les empêchent de se présenter à une évaluation abondent.

La distribution réussie de lignes directrices détaillées aux ophtalmologistes et aux optométristes à de nombreux endroits n'a pas eu d'effet important sur les pratiques de prise en charge de la rétinopathie diabétique et les recommandations relatives à l'évaluation et à l'examen ont été mal suivies. ${ }^{16,17,18,19}$

Au Canada, $32 \%$ seulement des patients qui ont le diabète de type 2 se conforment au

\section{(CSOO cont'd.)}

observateurs de chaque ordre (Ordre des médecins et chirurgiens de l'Ontario (OMCO) et Ordre des optométristes de l'Ontario (OOO)). Conformément à la recommandation du CCRPS, un cadre supérieur représentant le MSSLD assiste comme observateur aux travaux du CSOO, joue un rôle de conseiller auprès du Conseil et informe le ministère et le ministre au sujet des activités du Conseil.

Le CSOO vise finalement la prestation de soins oculovisuels de qualité, sécuritaires et accessibles par un professionnel le mieux placé pour le faire dans une région en cause de la province. Les temps d'attente diminueront ainsi, la qualité des soins s'améliorera et les résultats indésirables seront réduits au minimum. Les ordres professionnels indépendants (OMCO et OOO) continueront d'assurer la sécurité du public en réglementant les membres de leur profession. Par l'application des connaissances et le partage concerté de l'information sur les pratiques exemplaires, le Conseil pourra fournir de l'information précise à tous les participants, y compris le ministère, afin d'améliorer continuellement la prestation des soins oculovisuels en Ontario.

Le 23 septembre 2011, les membres du Conseil de la santé de l'œil de l'Ontario ont adopté à l'unanimité leurs premières lignes directrices sur la collaboration interprofessionnelle qui visent le soin des patients vivant avec le diabète sucré. Nous espérons que ces lignes directrices constituent un premier pas important vers l'amélioration de la prestation des soins de santé de l'œil aux patients vivant avec le diabète - et, éventuellement, à tous les Ontariens.

Respectueusement soumis,

DrThomas-A. Noël, BSC OD

Coprésident CSOO

Président de l'inscription

Membre du Conseil provincial

Ordre des optométristes de l'Ontario

Ottawa ON

SherifEl-Defrawy, MD, PhD, FRCSC

Coprésident CSOO

Professeur et directeur

Département d'ophtalmologie, Université Queen's

Ophtalmologiste en chef

Hôpitaux Hôtel Dieu et Kingston General

Kingston ON 
calendrier d'évaluation de la rétinopathie diabétique ${ }^{22}$ recommandé dans les lignes directrices de l'Association canadienne du diabète. ${ }^{20,21}$ Une étude portant sur les tendances de l'évaluation dans cinq provinces canadiennes a montré que $32 \%$ des personnes qui ont le diabète n'avaient pas subi d'examen des yeux au cours des deux dernières années et qu'une autre tranche de $32 \%$ n'avait jamais subi d'examen de dépistage de la DR. ${ }^{23}$

Les nombreux facteurs qui ont une incidence sur l'inobservation des lignes directrices recommandées comprennent le fait qu'on ne sait pas que la rétinopathie diabétique peut causer la cécité ou qu'une rétinopathie sévère peut être asymptomatique. ${ }^{24}$ Un accès limité aux professionnels des soins oculovisuels, en particulier en région éloignée, ${ }^{25,26,27}$ peut jouer un rôle important. La crainte des traitements au laser, la culpabilité suscitée par le mauvais contrôle à l'origine de la rétinopathie, l'inconvénient représenté par les traitements réguliers ${ }^{24}$ et la mobilité personnelle limitée à cause du mauvais état de santé général et d'une apathie autodéclarée ${ }^{28}$ peuvent aussi dissuader les patients de se présenter à leurs rendez-vous d'évaluation.

La recommandation du fournisseur de soins primaires au sujet de la nécessité d'un examen périodique des yeux constitue le prédicteur le plus important d'évaluation de dépistage de la rétinopathie diabétique et lorsqu'une telle recommandation est formulée, le taux d'évaluation s'améliore. ${ }^{29}$ Ainsi, tous les médecins et les autres professionnels de la santé devraient profiter des contacts avec des personnes qui ont le diabète pour en faire une occasion d'éducation au sujet de la nécessité d'une évaluation périodique de la vision et des facteurs de risque associés à la RD.

Les données probantes ${ }^{30}$ indiquent que la sensibilisation des patients à la rétinopathie diabétique, l'amélioration du rendement des fournisseurs et des pratiques, l'amélioration des processus infrastructuraux du système de santé afin de faciliter la participation des patients, les systèmes de rappels à envoyer aux patients et une meilleure approche des populations désavantagées peuvent améliorer considérablement les taux d'évaluation de la rétinopathie diabétique.

Toute stratégie ou tout programme d'évaluation a besoin de ressources suffisantes et doit avoir accès aux technologies de l'information pour assurer une couverture complète et l'observation des normes d'assurance de la qualité. $^{31}$

\section{Objectif}

Les présentes lignes directrices visent à coordonner les services des ophtalmologistes, optométristes, médecins de famille, médecins spécialistes, infirmières et infirmiers praticiens et autres professionnels de la santé dans la prise en charge des patients qui ont le diabète et à garantir ainsi l'utilisation la plus efficace des services de ces professionnels dans l'intérêt de la sécurité des patients, de la qualité des soins, de l'accessibilité et de l'efficacité des coûts.

\section{Rôles}

Fournisseurs de soins primaires :

Médecin de famille, médecin spécialiste, infirmière et infirmiers praticiens, autres professionnels de la santé

Pour prévenir les complications oculaires découlant du diabète, il faut commencer par déterminer la population à risque. Il incombe aux fournisseurs de soins primaires, $y$ compris les médecins de famille, de repérer les patients qui ont le diabète et ils jouent un rôle clé dans le soin et le traitement. En tant que coordonnateurs du soin des patients, les fournisseurs de soins primaires doivent référer le patient qui vient de recevoir un diagnostic de diabète de type 2 pour qu'il se soumette à une évaluation effectuée par un optométriste (ou un ophtalmologiste). Il faut repérer les patients qui ont passé la puberté avec le diabète de type 1 dans les cinq ans suivant le diagnostic.

Les patients en pédiatrie qui ont le diabète de type 1 devraient être référés pour un examen complet de la vue lorsqu'un enfant a 10 ans et a le diabète depuis au moins trois ans. Idéalement, un ophtalmologiste devrait effectuer ce premier examen. Lorsque le patient atteint l'âge de 13 ans, s'il n'a pas de rétinopathie, il doit être suivi par un optométriste (ou un ophtalmologiste) une fois par année.

Les médecins de famille doivent aussi veiller à ce que leurs patients établis qui ont le diabète de type 1 ou de type 2 mais sans rétinopathie soient évalués par un optométriste (ou un ophtalmologiste) une fois par 
année. Idéalement, chaque référence devrait être conjuguée à la détermination de la glycémie sanguine à jeun et des taux d'hémoglobine glycosylée.

\section{La tendance décrite ci-dessus des} références à un optométriste doit améliorer pour les patients l'accès à des services opportuns et uniformes de surveillance des maladies oculaires reliees au diabète. Le Conseil de la santé des yeux recommanderait que les premières références soient aiguillées vers un optométriste, mais le conseil n'a pas l'intention de restreindre l'accès direct à un ophtalmologiste en obligeant les patients à consulter d'abord un optométriste.

\section{Recommandations}

- Référer tout patient qui a dépassé l'âge de la puberté et qui a le diabète de type 1 dans les 5 ans suivant le diagnostic, pour une évaluation effectuée par un optométriste (ou un ophtalmologiste).

- Référer tout patient chez qui on vient de diagnostiquer un diabète de type 2 pour une évaluation par un optométriste (ou un ophtalmologiste). Le patient doit être vu dans les six mois suivant la référence.

- Référer tout patient en pédiatrie qui a le diabète de type 1 pour un examen complet de la vue lorsque l'enfant atteint l'âge de 10 ans, ou qu'il a le diabète depuis au moins trois ans. Idéalement, un ophtalmologiste devrait effectuer ce premier examen. Lorsque le patient atteint l'âge de 13 ans, un optométriste (ou un ophtalmologiste) devrait le suivre une fois par année.

- À chaque visite, il faut interroger un patient qui a le diabète au sujet de ses contacts avec un optométriste ou un ophtalmologiste pour assurer que le suivi est approprié.

- Comme on le verra plus loin dans ce document, l'optométriste et l'ophtalmologiste veilleront à ce que la visite périodique suivante de leur patient qui a le diabète soit arrangée et ils correspondront avec tous les médecins et les autres professionnels de la santé appropriés pour leur transmettre des mises à jour sur la santé oculaire du patient.

\section{Optométriste}

Les optométristes évalueront les patients en fonction des protocoles établis (voir plus loin) au sujet des complications oculaires du diabète et ils devraient fournir, au médecin de famille et au fournisseur de soins primaires, un rapport des constatations au cours du premier contact avec le patient et par la suite lorsque c'est indiqué sur le plan clinique. Il est utile de fournir une mise à jour annuelle si le patient est vu plus souvent. Lorsque l'on détecte une maladie des yeux d'origine diabétique, les optométristes devraient utiliser les critères généralement reconnus (voir plus loin) dans la prise en charge du patient ou lorsqu'ils réfèrent à un ophtalmologiste ou à un spécialiste des maladies de la rétine. La référence pour des soins subséquents devrait inclure un rapport à l'ophtalmologiste et au médecin de famille.

\section{Ophtalmologiste}

Les ophtalmologistes doivent évaluer et (au besoin) traiter les maladies oculaires d'origine diabétique afin de prévenir la perte de la vision, de la minimiser, ou de rétablir la vision. L'ophtalmologiste doit continuer de suivre les patients qui ont une maladie oculaire d'origine diabétique et qui risquent fort de perdre la vision. L'ophtalmologiste doit produire, lors du premier contact avec le patient et par la suite lorsque c'est indiqué sur le plan clinique, un rapport à l'intention du médecin de famille/ fournisseur de soins primaires et de l'optométriste. Il est utile de fournir une mise à jour annuelle si le patient est vu plus souvent.

Tous les professionnels doivent veiller à ce que leurs patients soient informés au sujet du diabète en général et de leur situation clinique en particulier.

\section{Évaluation initiale/ continue}

\section{Mise en place de l'évaluation des personnes qui ont le diabète de type 1 :}

Dans les cas de diabète de type 1 , la rétinopathie qui met la vision en danger est très rare au cours des cinq premières années ou avant la puberté. ${ }^{32} \mathrm{La}$ rétinopathie fait toutefois son apparition chez presque tous les patients qui ont le diabète de type 1 au cours des deux décennies qui suivent ${ }^{33}$ et il y a un lien solide entre la durée du diabète et l'apparition et la gravité de la $\mathrm{RD}^{34,35,36,37}$

Compte tenu des données probantes disponibles, l'évaluation de dépistage de la rétinopathie diabétique chez les personnes dont la puberté est terminée devrait commencer dans les cinq ans suivant le diagnostic. 
Dans le cas des personnes qui n'ont pas atteint la puberté, il faudrait mettre en place l'évaluation à l'âge de 10 ans ou dans les trois ans suivant le diagnostic, selon l'échéance la plus rapprochée.

\section{Mise en place de l'évaluation des personnes qui ont le diabète de type 2 :}

La durée du diabète constitue le facteur de risque le plus solide relié à l'apparition de la RD. ${ }^{38,39,40,41,42} \mathrm{Le}$ risque de RD est continu et il n'y aucun seuil évident relié à la glycémie ou à la tension artérielle. ${ }^{75}$

Au moment où l'on diagnostique le diabète, jusqu'à $3 \%$ des patients qui ont le diabète et ont plus de 30 ans ont un œdème maculaire significatif sur le plan clinique (OMSC) ou une $\mathrm{RD}$ à risque élevé. ${ }^{43,44}$ Après 10 ans, $7 \%$ des personnes qui ont le diabète ont une rétinopathie, pourcentage qui passe à $90 \%$ après 25 ans. On a découvert la maladie proliférante chez $20 \%$ des patients qui avaient le diabète depuis 20 ans. ${ }^{45} \mathrm{Il}$ a été démontré que la prévalence de la $\mathrm{RD}$ est plus faible chez les patients qui reçoivent un diagnostic de diabète après l'âge de 70 ans et les patients qui ont une $\mathrm{RD}$ avaient le diabète depuis beaucoup plus longtemps ( 5,0 ans) en moyenne que ceux qui n'ont pas la $\mathrm{RD}\left(3,5\right.$ ans). ${ }^{46}$

Il s'écoule sept ans entre l'apparition des symptômes et le diagnostic chez les patients qui ont le diabète de type 2 . Compte tenu de ce facteur et des renseignements qui précèdent, l'évaluation de dépistage de la rétinopathie chez les patients qui ont le diabète de type 2 devrait commencer au moment où le diagnostic est posé.
Intervalles entre les évaluations des personnes qui ont le diabète :

On signale depuis 1985 des taux moins élevés d'évolution en RDP et de perte sévère de la vision causée par la RD, ce qui peut être attribuable à une sensibilisation accrue aux facteurs de risque de rétinopathie, à la détermination et aux soins plus précoces des patients qui ont une rétinopathie ainsi qu'à une meilleure prise en charge de la glycémie, de la tension artérielle et des lipides sériques. ${ }^{47}$

\section{Diabète de type 1}

L'étude prospective sur les complications réalisée par EURODIAB a révélé que la durée du diabète, l'âge à l'apparition des symptômes avant 12 ans et le contrôle du métabolisme constituaient des prédicteurs importants de l'évolution, même lorsque l'on tient compte de la présence d'une rétinopathie de base. ${ }^{48}$

\section{Recommandations Spécifiques}

\section{AUCUNE RÉTINOPATHIE}

\section{Diabète de type 1}

Les données probantes disponibles indiquent qu'une évaluation annuelle doit être effectuée par un optométriste (ou un ophtalmologiste, ou au moyen d'un dépistage par télémédecine si ces médecins ne sont pas accessibles).

\section{Diabète de type 2}

Lorsqu'il n'y a pas de RD, on n'établit aucun lien entre des évaluations aux 19 à 24 mois plutôt qu'aux
12 à 18 mois et un risque accru de rétinopathie à présenter ${ }^{49}$ et il a été démontré que le dépistage biennal est sécuritaire et efficace, car personne n'a vu son état évoluer de l'absence de rétinopathie à la présence d'une rétinopathie qui met la vision en danger en moins de deux ans. ${ }^{50}$ Cette approche réduit de plus de $25 \%$ le nombre des évaluations, ce qui réduit considérablement les coûts de la santé et les pressions imposées sur les ressources et évite des examens inutiles aux patients qui ont le diabète. ${ }^{51}$ On établit toutefois un lien entre des intervalles de plus de 24 mois entre les évaluations de dépistage et un risque accru de $\mathrm{RD}$ mettant la vision en danger. ${ }^{49} \mathrm{La}$ grande préoccupation, c'est toutefois que si on laisse tomber les examens annuels, les patients ne receuront pas le suivi approprié. C'est particulièrement vrai dans le cas des personnes qui ont un accès médiocre aux soins. Comme l'examen annuel constitue la norme de diligence en vigueur pour les personnes qui ont le diabète de type 1, ce sera ce que recommanderont les présentes lignes directrices dans le cas des patients qui ont le diabète de type 2. On peut suggérer un suivi biennal dans le cas des patients sur lesquels on peut compter pour qu'ils reconnaissent le besoin d'un rappel après 24 mois ou dans celui des bureaux qui peuvent rappeler les patients efficacement après deux ans.

Une évaluation annuelle des patients qui ont le diabète de type 2 et n'ont pas de rétinopathie pourrait être effectuée par un optométriste (ou un ophtalmologiste, ou au moyen d'un dépistage par télémédecine si ces médecins ne sont pas accessibles). 


\section{FEMMES ENCEINTES QUI ONT LE DIABÈTE}

Avant d'essayer de devenir enceintes, les femmes qui ont le diabète de type 1 ou 2 doivent se soumettre à une évaluation ophtalmique effectuée par un optométriste ou un ophtalmologiste. Il faut procéder à des évaluations répétitives au cours du premier trimestre et de nouveau, au besoin, au cours de l'année qui suit l'accouchement. ${ }^{76}$ Cette ligne directrice ne s'applique pas aux femmes chez lesquelles le diabète gestationnel fait son apparition parce qu'elle ne sont pas à risque accru de rétinopathie diabétique.

\section{RÉTINOPATHIE MINIME : RDNP légère}

- Plusieurs microanévrismes

- Acuité visuelle de $6 / 6$ ou mieux (avant qu'il y ait une autre cause connue de baisse de la vision)

Suivi annuel des patients qui ont une RDNP légère par un optométriste (ou un ophtalmologiste, ou par télémédecine si ces médecins ne sont pas accessibles).

\section{RÉTINOPATHIE MODÉRÉE : RDNP modérée}

v Hémorragies intrarétiniennes

- Exsudats durs

- Infarctus de la couche des fibres nerveuses/exsudats cotonneux

Il faut envisager de référer un patient qui a une RDNP modérée à un ophtalmologiste (ou à un spécialiste de la rétine) s’il y a la moindre préoccupation au sujet de l'OMD, de l'OMSC ou de toute autre maladie traitable. Les patients qui ont une RDNP modérée doivent être évalués par un professionnel des soins oculovisuels (optométriste ou ophtalmologiste) au moins aux six mois.

\section{RÉTINOPATHIE SÉVÈRE : RDNP sévère}

La RDNP inclut toutes les caractéristiques de la RDNP et n'importe laquelle des suivantes :

- Hémorragies intrarétiniennes ( $\geq 20$ dans chacun des quatre quadrants)

- Nodules veineux (deux quadrants ou plus)

- Rétrécissement des artérioles

- Anomalies microvasculaires intrarétiniennes - AMIR (un quadrant ou plus)

La RDNP très sévère s'entend de la présence de deux des critères indiquant une RDNP sévère.

Référence à un spécialiste de la rétine (ou ophtalmologiste) pour traitement possible. Évaluation par un ophtalmologiste aux deux à quatre mois. Une fois stabilisé, le patient doit être suivi par un optométriste ou un ophtalmologiste (ou un spécialiste de la rétine) afin qu'il y ait évaluation au moins aux six mois.

\section{đEDÈME MACULAIRE DIABÉTIQUE : DME, OMSC}

L'œdème maculaire significatif sur le plan clinique (OMSC) se définit ainsi ${ }^{74}$ :

- Épaississement de la rétine au niveau de la fovéa ou à moins de 500 microns de celle-ci

- Exsudats durs au niveau de la fovéa ou à moins de 500 microns de celle-ci (si la rétine adjacente est épaissie)

- Épaississement de la rétine d'un diamètre du disque ou plus ou s'il se produit à moins d'un diamètre du disque de la fovéa.

Référence à un spécialiste de la rétine (ou ophtalmologiste) pour traitement (laser, IVI). Suivi par l'ophtalmologiste traitant jusqu'à ce que l'EMD se soit stabilisé ou résorbé. Une fois stabilisé, le patient doit être suivi par un optométriste ou un ophtalmologiste (ou un spécialiste de la rétine) afin qu'il y ait évaluation au moins aux six mois.

\section{RÉTINOPATHIE DIABÉTIQUE PROLIFÉRANTE : RDP}

- Néovascularisation du disque NVD

— Néovascularisation ailleurs NVA

- Hémorragie du vitré/prérétinienne

- Néovascularisation de l'iris - NVI (néovascularisation du segment antérieur) 
Référence à un spécialiste de la rétine (ou ophtalmologiste) pour traitement (laser, IVI, vitrectomie). Suivi par l'ophtalmologiste traitant jusqu'à la régression. Une fois stabilisé, le patient doit être suivi par un optométriste ou un ophtalmologiste (ou un spécialiste de la rétine) afin que l'évaluation ait lieu au moins aux six mois.

\section{Outils d'évaluation}

L'évaluation du patient à la fois par l'ophtalmologiste et par l'optométriste comprend un examen complet de toutes les structures oculaires et un commentaire sur toute complication oculaire associée au diabète, plutôt qu’à la rétinopathie diabétique seulement. Un examen clinique visant à détecter et évaluer la RD et sa gravité peut être effectué par biomicroscopie à lampe à fente, ophtalmoscopie ou photographie rétinienne. L'examen devrait inclure la mesure de l'acuité visuelle et les pupilles devraient normalement être dilatées pour l'examen du fond de l'œil. Les examinateurs doivent faire preuve d'une sensibilité et d'une spécificité adéquates dans l'exécution de toutes les évaluations. La sensibilité minimale dans le cas de la $\mathrm{RD}$ a été fixée à $80 \% \%^{53,54}$ ou, dans le cas de l'examen répété qui détecterait une $\mathrm{RD}$ non repérée au cours d'examens antérieurs, à $60 \% .{ }^{55}$ Des niveaux de spécificité de 90 à $95 \%$ et des taux de défaillance technique de 5 à $10 \%$ sont jugés appropriés. ${ }^{54}$

\section{Biomicroscopie}

La biomicroscopie à lampe à fente effectuée au moyen d'une lampe d'examen du fond de l'œil sans contact après dilatation des pupilles constitue la norme de pratique courante dans le cas de la détection de la RD (sensibilité de $87,4 \%$ et spécificité de $94,4 \%$ ) et est préférée à l'ophtalmoscopie directe, moins sensible et plus variable, même lorsqu'elle est effectuée par un examinateur chevronné (sensibilité de 56 à $98 \%$, spécificité de 62 à $100 \%) .{ }^{56}$ La formation devrait donner aux examinateurs une précision diagnostique suffisante et une sensibilité et une spécificité adéquates. ${ }^{54,57}$ La photographie rétinienne à champ unique ou la tomographie par cohérence optique ne remplacent pas l'examen approprié de la rétine avec pupilles dilatées.

\section{Photographie rétinienne}

La photographie stéréoscopique du fond de l'œil à sept champs évaluée par un examinateur qui a reçu de la formation constitue «l'étalon-or » de la détection de la RD et a été utilisée dans la plupart des essais cliniques d'envergure réalisés dans ce domaine. Cette technique coûte toutefois cher en temps et en argent et sert rarement dans la pratique de routine. La photographie rétinienne à champ unique peut être utile aux fins de la documentation et du suivi comme élément d'un examen complet effectué par un optométriste ou un ophtalmologiste.

\section{Télémédecine}

La photographie rétinienne numérique sert de plus en plus dans le dépistage de la RD. Elle ne remplace pas un examen complet de l'œil, mais lorsqu'il n'y a pas d'optométriste ou d'ophtalmologiste disponible, des données probantes de niveau I indiquent qu'elle peut servir au dépistage de la rétinopathie diabétique. Les patients qui ont une rétinopathie révélée par cette méthode doivent être référés à un optométriste ou à un ophtalmologiste pour l'évaluation plus poussée et prise en charge. . $^{5,59,60,61,62,63}$

L'imagerie du fond de l'œil offre l'avantage supplémentaire d'être perçue par les patients comme une ressource d'éducation précieuse. ${ }^{24}$ Elle peut être effectuée avec pupilles dilatées ou au moyen de caméras non mydriatiques à travers des pupilles non dilatées. ${ }^{64} \mathrm{La}$ technologie choisie, ainsi que le nombre de champs de prise de vue, auront un effet sur la sensibilité du dépistage. ${ }^{65}$

\section{Angiographie à la fluorescéine (AF)}

L'angiographie à la fluorescéine ne joue aucun rôle dans le dépistage de la RD, mais elle est essentielle dans un cas de maladie au stade tardif pour détecter et circonscrire l'ischémie rétinienne. Cet examen effractif comporte un risque inhérent mais faible d'effets secondaires importants, dont certains sont bénins et provisoires et d'autres, sévères (comme l'anaphylaxie ou l'arrêt cardiaque).

\section{Tomographie par cohérence optique (TCO)}

La tomographie par cohérence optique est une technique non effractive et sans contact qui produit des images transversales de la rétine et du disque optique semblables à des coupes histologiques. Sa résolution axiale est de $5 \mu \mathrm{m}$ dans le cas des instruments plus modernes et elle produit des données qualitatives et quantitatives qui permettent 
d'établir une bonne corrélation avec la stéréophotographie du fond de l'œil ou la biomicroscopie afin de diagnostiquer l'œdème maculaire diabétique. Cette technique offre une bonne reproductibilité et mesure avec précision l'épaisseur de la rétine. ${ }^{67,68}$

La TCO semble utile pour détecter l'épaississement maculaire aux stades précoces de la rétinopathie diabétique chez les patients qui ont une rétinopathie et ne présentent aucun signe clinique d'œdème maculaire, ce qui permet d'effectuer un suivi plus rapproché pour détecter l'EMD au stade précoce. ${ }^{69,70} \mathrm{La}$ TCO n'aide toutefois pas à prédire dans quel œil atteint d'EMD sous-clinique le problème évoluera en EMD cliniquement significative. ${ }^{71}$

La TCO est une méthode qualitative et quantitative efficace qui permet de détecter l'épaississement maculaire précoce et de suivre l'évolution ou la régression de l'œdème maculaire pendant le traitement. On a intégré cette technique comme mesure de routine dans de nombreuses études en cours portant sur de nouveaux traitements de la RD.

Les données courantes indiquent qu'il y a peu de raison de soumettre à une TCO de routine des personnes diabétiques qui n'ont pas de rétinopathie ou qui ont une rétinopathie diabétique légère à modérée lorsque l'examen clinique ne révèle pas d'œdème maculaire. ${ }^{72} \mathrm{Il}$ faudrait toutefois envisager sérieusement la TCO face à des changements de la structure maculaire ou à tout changement inexpliqué de l'acuité la mieux corrigée.

\section{Conclusion}

Il est essentiel de coordonner les ressources de la santé dans le soin et le traitement des patients à risque de complications oculaires causées par le diabète. L'évaluation optométrique opportune des patients diabétiques qui viennent de recevoir un diagnostic permettra de repérer les patients à risque de maladie oculaire diabétique. L'intervention et le traitement précoces des maladies oculaires grâce à la référence appropriée et opportune pour des soins ophtalmologistes aideront à préserver la qualité de la vision des patients qui ont le diabète. Des lignes directrices interprofessionnelles et des critères de gestion et de référence généralement reconnus permettront d'assurer une coordination appropriée des soins et l'utilisation la plus efficace des ressources en professionnels de la santé.

\section{Références}

1. Lipscombe L. Hux J. Trends in diabetes prevalence, incidence and mortality in Ontario, Canada 1995-2005: a population-based study. Lancet. 2007; 369 (9563): 750-756.

2. Klein R, Klein BEK. Vision disorders in diabetes. Dans : National Diabetes Data Group. Diabetes in America, 2nd ed. National Institutes of Health. 1995; (95-1468): 293-338.

3. Javitt JC, Canner JK, Sommer A. Cost effectiveness of current approaches to the control of retinopathy in type I diabetes. Ophthalmology. 1989; 96: 255264.

4. Ferris F. How effective are treatments for diabetic retinopathy? JAMA. 1993. 269: 1290-1291.

5. CDA. http://www.diabetes. ca/documents/about-diabetes/ PrevalanceandCostFR_09.pdf

6. Bowyer NK. Diabetic Retinal Changes Linked to Amputation. Review of Optometry. 2003. December.
7. Moss SE, Klein R, Klein BEK, Wong TY. Retinal vascular changes and 20 year incidence of lower extremity amputations in a cohort with diabetes. Arch Intern Med. 2003. 163: 2505-2510.

8. INCA. Maladies oculaires. Diabète et affection des yeux. Toronto : INCA. http://www.diabetes. $\mathrm{ca} /$ documents/about-diabetes/ PrevalanceandCostFR_09.pdf

9. Commonwealth of Australia. NHMRC Clinical Practice Guidelines (1997) Management of diabetic retinopathy, Commonwealth of Australia Publication No 2142, Commonwealth Department of Health and Family Services. 1997. Canberra.

10. Wang F, Javitt JC. Eye care for elderly Americans with diabetes mellitus. Failure to meet current guidelines. Ophthalmology. 1997. 103. 1744-1750.

11. A Clear Vision. Solutions to Canada's Vision Loss Crisis, Toronto ON : Canterbury Communications; 2004. http://www.costofblindness.org

12. Ministère de la Santé et des Soins de longue durée de l'Ontario. Groupe de travail sur le diabète. Rapport présenté au ministère de la Santé et des Soins de longue durée. 2004.http://www. health.gov.on.ca/french/publicf/pubf/ ministry_reportsf/diabetes_taskforcef/ diabetes_taskforcef.html

13. Kahn HA, Hillier R. Blindness caused by diabetic retinopathy. Am J Ophthalmol. 1974. 78:58-67.

14. International Council of Ophthalmology. International Standards: For Vision, Eye Care and Ophthalmology. http://www.icoph. org/standards/index.html

15. Sloan FA, Grossman DS, Lee PP. Effects of receipt of guidelinerecommended care on onset of diabetic retinopathy and its progression. Ophthalmology. 2009 Aug;116(8):151521, 1521.e1-3.

16. Schoenfeld ER, Greene JM, Wu SY, Leske MC. Patterns of adherence to diabetes vision care guidelines: baseline findings from the Diabetic Retinopathy Awareness Program. Ophthalmology Mars 2001;108(3):563-71.

17. Lee SJ, Livingston PM, Harper CA, McCarty CA, Taylor HR, Keeffe 
JE.Compliance with recommendations from a screening programme for diabetic retinopathy.Aust N Z J Ophthalmol. Juinaoût 1999;27(3-4):187-9.

18. Lee SJ, Sicari C, Harper CA, Livingston PM, McCarty CA, Taylor HR, Keeffe JE.Examination compliance and screening for diabetic retinopathy: a 2-year follow-up study.Clin Experiment Ophthalmol. Juin 2000;28(3):149-52. PMID: 10981784

19. McCarty CA, Wright S, McKay R, Taylor KI, Keeffe JE; Working Group on Evaluation of NHMRC Diabetic Retinopathy Guidelines. Changes in management of diabetic retinopathy by Australian ophthalmologists as a result of the NHMRC clinical guidelines. Clin Experiment Ophthalmol. Août 2001;29(4):230-4

20. Clark HD, van Walraven C, Code C, Karovitch A, Keely E. Diabetes Care. Did publication of a clinical practice guideline recommendation to screen for type 2 diabetes in women with gestational diabetes change practice? Fév. 2003;26(2):265-8.

21. Woo V; CDA 2008 Clinical Practice Guidelines Steering Committee. Medical management of hyperglycemia in type 2 diabetes: a consensus algorithm for the initiation and adjustment of therapy: a consensus statement of the American Diabetes Association and the European Association for the Study of Diabetes: response to Nathan et al. Diabetes Care. 2009 Mar;32(3):e34; author reply e37-8. Aucun résumé disponible.

22. Sloan FA, Brown DS, Carlisle ES, Picone GA, Lee PP. Monitoring visual status: why patients do or do not comply with practice guidelines. Health Serve Res. Oct. 2004;39(5):1429-48.PMID: 15333116

23. Boucher MC, Earaches G, GarciaSalinas R, Kearny A, Maberley D, Olivier S, Oh M, Stockl F. Teleophthalmology screening for diabetic retinopathy through mobile imaging units within Canada. Journal canadien d'opthalmologie. Déc. 2008;43(6): 658-68.

24. Lewis K, Patel D, Yorston D, Charteris D. A qualitative study in the United Kingdom of factors influencing attendance by patients with diabetes at ophthalmic outpatient clinics. Ophthalmic Epidemiol. Nov.-déc. 2007;14(6):375-80.PMID: 18161611

25. Maberley DA, Koushik A, Cruess AF. Factors associated with missed eye examinations in a cohort with diabetes. Can J Public Health. Mai-juin 2002;93(3):229-32.PMID: 12050993

26. Mukamel DB, Bresnick GH, Wang Q, Dickey CF. Barriers to compliance with screening guidelines for diabetic retinopathy. Ophthalmic Epidemiol. Mars 1999;6(1):61-72.

27. Leese GP, Boyle P, Feng Z, EmslieSmith A, Ellis JD. Screening uptake in a well-established diabetic retinopathy screening program: the role of geographical access and deprivation. Diabetes Care. Nov. 2008;31(11):2131-5. Epub 26 août 2008.PMID: 18728235

28. Puent BD, Nichols KK. Patients' perspectives on noncompliance with diabetic retinopathy standard of care guidelines. Optometry. Nov. 2004;75(11):709-16.

29. Dervan E, Lillis D, Flynn L, Staines A, O'Shea D. Factors that influence the patient uptake of diabetic retinopathy screening. Déc. 2008;177(4):303-8. Epub 19 juil. 2008.

30. Zhang X, Norris SL, Saadine J, Chowdhury FM, Horsley T, Kanjilal $\mathrm{S}$, Mangione CM, Buhrmann R. Effectiveness of interventions to promote screening for diabetic retinopathy. Am J Prev Med. Oct. 2007;33(4):318-35. Review.PMID: 17888859

31. Goldstein DE, Blinder KJ, Ide CH, Wilson RJ, Wiedmeyer HM, Little RR, England JD, Eddy M, Hewett JE, Anderson SK. Glycemic control and development of retinopathy in youth-onset insulin-dependent diabetes mellitus. Results of a 12-year longitudinal study. Ophthalmology. Août 1993;100(8):1125-31; discussion 1131-2.

32. Orchard TJ, Dorman JS, Maser RE, Becker DJ, Drash AL, Ellis D, LaPorte RE, Kuller LH.Prevalence of complications in IDDM by sex and duration. Pittsburgh Epidemiology of Diabetes Complications Study II. Diabetes. Sept. 1990;39(9):1116-24.

33. ibid.

34. d'Annunzio G, Malvezzi F, Vitali L, Barone C, Giacchero R, Klersy C, Zanette S, Lorini R. Diabet Med. A 3-19-year follow-up study on diabetic retinopathy in patients diagnosed in childhood and treated with conventional therapy. Nov. 1997;14(11):951-8.

35. Younis N, Broadbent DM, Vora JP, Harding SP; Liverpool Diabetic Eye Study. Incidence of sight-threatening retinopathy in patients with type 2 diabetes in the Liverpool Diabetic Eye Study: a cohort study. Lancet. 18 janv. 2003;361(9353):195-200.

36. Klein R, Klein BE, Moss SE, Cruickshanks KJ. The Wisconsin Epidemiologic Study of diabetic retinopathy. XIV. Ten-year incidence and progression of diabetic retinopathy. Arch Ophthalmol. Sept. 1994;112(9):1217-28.

37. ibid.

38. Cohen O, Norymberg K, Neumann E, Dekel H. Complication-free duration and the risk of development of retinopathy in elderly diabetic patients. Arch Intern Med. 23 mars 1998;158(6):641-4.

39. Zhang X, Norris SL, Saadine J, Chowdhury FM, Horsley T, Kanjilal $\mathrm{S}$, Mangione CM, Buhrmann R. Effectiveness of interventions to promote screening for diabetic retinopathy. Am J Prev Med. Oct. 2007;33(4):318-35. Review.

40. Klein R, Klein BE, Moss SE, Cruickshanks KJ. The Wisconsin Epidemiologic Study of diabetic retinopathy. XIV. Ten-year incidence and progression of diabetic retinopathy. Arch Ophthalmol. Sept. 1994;112(9):1217-28.

41. Mitchell P. Development and progression of diabetic eye disease in Newcastle (1977-1984): rates and risk factors. Aust N Z J Ophthalmol. Fév. 1985;13(1):39-44.

42. [Aucun auteur indiqué] Tight blood pressure control and risk of macrovascular and microvascular 
complications in type 2 diabetes: UKPDS 38. UK Prospective Diabetes Study Group. BMJ. 12 sept. 1998;317(7160):703-13. Erratum in: BMJ 2 janv. 1999;318(7175):29.

43. Klein R, Moss SE, Klein BE. New management concepts for timely diagnosis of diabetic retinopathy treatable by photocoagulation. Diabetes Care. Sept.-oct. 1987;10(5):633-8.

(44) Klein BE, Moss SE, Klein R. Longitudinal measure of glycemic control and diabetic retinopathy. Diabetes Care. Mai-juin 1987;10(3):2737.

45. Bhavsar AR. Diabetic retinopathy. The diabetes eye exam initiative. Minn Med. Juin 2002;85(6):46-7. Aucun résumé disponible.

46. Cahill M, Halley A, Codd M, O'Meara N, Firth R, Mooney D, Acheson RW. Prevalence of diabetic retinopathy in patients with diabetes mellitus diagnosed after the age of 70 years. $\mathrm{Br} \mathrm{J}$ Ophthalmol. Mars 1997;81(3):218-22.

47. Wong TY, Mwamburi M, Klein R, Larsen M, Flynn H, Hernandez-Medina M, Ranganathan G, Wirostko B, Pleil A, Mitchell P. Rates of progression in diabetic retinopathy during different time periods: a systematic review and meta-analysis. Diabetes Care. Déc. 2009;32(12):2307-13.

48. Porta M, Sjoelie AK, Chaturvedi N, Stevens L, Rottiers R, Veglio M, Fuller JH; EURODIAB Prospective Complications Study Group. Risk factors for progression to proliferative diabetic retinopathy in the EURODIAB Prospective Complications Study. Diabetologia. Déc. 2001;44(12):2203-9.

49. Misra A, Bachmann MO, Greenwood RH, Jenkins C, Shaw A, Barakat O, Flatman M, Jones CD. Trends in yield and effects of screening intervals during 17 years of a large UK community-based diabetic retinopathy screening programme. Diabet Med. Oct. 2009;26(10):1040-7.

50. Mitchell P. The prevalence of diabetic retinopathy: a study of 1300 diabetics from Newcastle and the Hunter Valley. Aust J Ophthalmol. Août 1980;8(3):241-6.

51. Olafsdóttir E, Stefánsson E. Biennial eye screening in patients with diabetes without retinopathy: 10- year experience. Br J Ophthalmol. Déc. 2007;91(12):1599-601. Epub 12 juil. 2007.

52. Diabetes Control and Complications Trial/Epidemiology of Diabetes Interventions and Complications (DCCT/EDIC) Research Group, Nathan DM, Zinman B, Cleary PA, Backlund JY, Genuth S, Miller R, Orchard TJ. Modern-day clinical course of type 1 diabetes mellitus after 30 years' duration: the diabetes control and complications trial/epidemiology of diabetes interventions and complications and Pittsburgh epidemiology of diabetes complications experience (1983-2005). Arch Intern Med. 27 juil. 2009;169(14):1307-16.

53. Moss SE, Klein R, Kessler SD, Richie KA. Comparison between ophthalmoscopy and fundus photography in determining severity of diabetic retinopathy. Ophthalmology. Janv. 1985;92(1):62-7.

54. Hutchinson A, McIntosh A, Peters J, O'Keeffe C, Khunti K, Baker R, Booth A. Diabet Med. Effectiveness of screening and monitoring tests for diabetic retinopathy--a systematic review. Juil. 2000;17(7):495-506.

55. Javitt JC, Aiello LP, Bassi LJ, Chiang YP, Canner JK. Detecting and treating retinopathy in patients with type I diabetes mellitus. Savings associated with improved implementation of current guidelines. American Academy of Ophthalmology. Ophthalmology. Oct. 1991;98(10):1565-73; discussion 1574.

56. Leese G, Broadbent D, Harding S, Vora J. Screening for diabetic retinopathy. Approaching 90\% sensitivity with new techniques. BMJ. 4 nov. 1995;311(7014):1230-1.

57. Gibbins RL, Owens DR, Allen JC, Eastman L. Practical application of the European Field Guide in screening for diabetic retinopathy by using ophthalmoscopy and $35 \mathrm{~mm}$ retinal slides. Diabetologia. Janv. 1998;41(1):5964.

58. Vujosevic S, Benetti E, Massignan F, Pilotto E, Varano M, Cavarzeran F, Avogaro A, Midena E. Screening for diabetic retinopathy: 1 and 3 nonmydriatic 45-degree digital fundus photographs vs 7 standard early treatment diabetic retinopathy study fields. Am J Ophthalmol. Juil. 2009;148(1):111-8. Epub 5 mai 2009.

59. Williams GA, Scott IU, Haller JA, Maguire AM, Marcus D, McDonald HR. Single-field fundus photography for diabetic retinopathy screening: a report by the American Academy of Ophthalmology. Ophthalmology. Mai 2004;111(5):1055-62. Review.

60. Boucher MC, Gresset JA, Angioi K, Olivier S. Effectiveness and safety of screening for diabetic retinopathy with two nonmydriatic digital images compared with the seven standard stereoscopic photographic fields. Journal canadien d'ophtalmologie. Déc. 2003;38(7):557-68.

61. Perrier M, Boucher MC, Angioi K, Gresset JA, Olivier S. Comparison of two, three and four 45 degrees image fields obtained with the Topcon CRW6 nonmydriatic camera for screening for diabetic retinopathy. Journal canadien d'ophtalmologie. Déc. 2003;38(7):56974.

62. Fransen SR, Leonard-Martin TC, Feuer WJ, Hildebrand PL; Inoveon Health Research Group. Clinical evaluation of patients with diabetic retinopathy: accuracy of the Inoveon diabetic retinopathy-3DT system. Ophthalmology. Mars 2002;109(3):595601.

63. Patra S, Gomm EM, Macipe M, Bailey C. Interobserver agreement between primary graders and an expert grader in the Bristol and Weston diabetic retinopathy screening programme: a quality assurance audit. Diabet Med. Août 2009;26(8):820-3.

64. Whited JD, Datta SK, Aiello LM, Aiello LP, Cavallerano JD, Conlin PR, Horton MB, Vigersky RA, Poropatich RK, Challa P, Darkins AW, Bursell SE. A modeled economic analysis of a digital tele-ophthalmology system as used by three federal health care agencies for detecting proliferative diabetic retinopathy. Telemed J E Health. Déc. 2005;11(6):641-51.

65. Baeza M, Orozco-Beltrán D, Gil-Guillen VF, Pedrera V, Ribera MC, Pertusa S, Merino. J.Int J Clin Pract. Screening for 
sight threatening diabetic retinopathy using non-mydriatic retinal camera in a primary care setting: to dilate or not to dilate? Mars 2009;63(3):433-8.

66. Pugh JA, Jacobson JM, Van Heuven WA, Watters JA, Tuley MR, Lairson DR, Lorimor RJ, Kapadia AS, Velez R. Screening for diabetic retinopathy. The wide-angle retinal camera. Diabetes Care. Juin 1993;16(6):889-95.

67. Virgili G, Menchini F, Dimastrogiovanni AF, Rapizzi E, Menchini U, Bandello F, Chiodini RG. Optical coherence tomography versus stereoscopic fundus photography or biomicroscopy for diagnosing diabetic macular edema: a systematic review. Invest Ophthalmol Vis Sci. Nov. 2007;48(11):4963-73. Review.

68. Browning DJ, Glassman AR, Aiello LP, Bressler NM, Bressler SB, Danis RP, Davis MD, Ferris FL, Huang SS, Kaiser PK, Kollman C, Sadda S, Scott IU, Qin H; Diabetic Retinopathy Clinical Research Network. Optical coherence tomography measurements and analysis methods in optical coherence tomography studies of diabetic macular edema. Ophthalmology. Août 2008;115(8):1366-71, 1371.e1.

69. Koleva-Georgieva DN, Sivkova NP. Optical coherence tomography for the detection of early macular edema in diabetic patients with retinopathy. Folia Med (Plovdiv). Janv.-mars 2010; 52(1):40-8.

70. Hannouche RZ, Avila MP. Retinal thickness measurement and evaluation of natural history of the diabetic macular edema through optical coherence tomography. Arq Bras Oftalmol. Juil.-août 2009;72(4):433-8.

71. Browning DJ, Fraser CM, Propst BW. The variation in optical coherence tomography-measured macular thickness in diabetic eyes without clinical macular edema. Am J Ophthalmol. Mai 2008;145(5):889-93. Epub 10 mars 2008 .

72. Browning DJ, Glassman AR, Aiello LP, Bressler NM, Bressler SB, Danis RP, Davis MD, Ferris FL, Huang SS, Kaiser PK, Kollman C, Sadda S, Scott IU, Qin H; Diabetic Retinopathy Clinical Research Network. Optical coherence tomography measurements and analysis methods in optical coherence tomography studies of diabetic macular edema. Ophthalmology. Août 2008;115(8):1366-71, 1371.e1.

73. ETDRS Study Group. Treatment techniques and clinical guidelines for photocoagulation of diabetic macular edema (ETDRS Report Number 2). Ophthalmology. 1987; 94(7): 761-74

74. Stratton IM, et al. Association of glycaemia with macrovascular and microvascular complications of type 2 diabetes (UKPDS 35): prospective observational study. BMJ 2000; 321: 405-12.
75. The Diabetes Control and Complications Trial Research Group. Effect of pregnancy on microvascular complications in the Diabetes Control and Complications Trial. Diabetes Care 2000; 23: 1084-91.

76. The Diabetes Control and Complications Trial Research Group. Early worsening of retinopathy in the Diabetes Control and Complications Trial. Arch Opthalmol. 1998; 116: 874 86.

\section{Annexe : Échelle de gravité de la rétinopathie diabétique (RD)}

\section{Aucune rétinopathie diabétique apparente}

\section{Rétinopathie diabétique non proliférante} (RDNP

- RDNP moyenne à modérée microanévrismes, hémorragies intrarétiniennes, exsudats durs, anomalies de la zone vasculaire de la fovéa

- RDNP modérée à sévère - exsudats cotonneux, nodules veineux, anomalies microvasculaires intrarétiniennes (AMIR)

- RDNP sévère (règle 4-2-1) - n'importe lequel des symptômes suivants : hémorragies intrarétiniennes sévères $(>20)$ dans chacun des quatre quadrants, nodules veineux dans deux quadrants ou plus; AMIR évidente dans un quadrant ou plus

- RDNP très sévère - deux des critères ci-dessus

Rétinopathie diabétique proliférante (RDP) - au moins un des symptômes suivants :

- Neovascularization of the disc-NVD

- Néovascularisation du disque - NVD (en particulier dont la taille dépasse un diamètre du disque)

- Néovascularisation ailleurs - NVA
- Hémorragie dans le vitré/prérétinienne

- Néovascularisation de l'iris - NVI (néovascularisation du segment antérieur)

Fdème maculaire (diabétique) cliniquement significatif (EMCS)

- tout épaississement rétinien situé à moins de 500 micromètres du centre de la macule (fovéa)

- épaississement rétinien dont la taille est d'au moins un diamètre papillaire mais dont n'importe quelle partie se situe à moins d'un diamètre du centre de la macule (fovéa)

- exsudats durs situés à moins de 500 micromètres du centre de la macule (fovéa) avec épaississement de la rétine adjacente

Il importe de signaler que les exsudats durs sont un signe d'œdème maculaire en cours ou antérieur. L'EMCS peut être focal (fuite provenant de microanévrismes ou d'AMIR) ou diffus (fuite provenant du lit capillaire sousjacent). L'EMCS constitue la cause la plus répandue de baisse de la vision et de cécité chez les patients qui ont le diabète et peut se manifester en même temps que la rétinopathie diabétique à n'importe quel stade. 